\title{
Fiat and Bona Fide Boundaries
}

\author{
BARRY SMITH \\ State University of New York at Buffalo, Buffalo, NY
}

ACHILLE C. VARZI

Columbia University, New York, NY

\begin{abstract}
There is a basic distinction, in the realm of spatial boundaries, between bona fide boundaries on the one hand, and fiat boundaries on the other. The former are just the physical boundaries of old. The latter are exemplified especially by boundaries induced through human demarcation, for example, in the geographic domain. The classical metaphysical problems connected with the notions of adjacency, contact, separation, and division can be resolved in an intuitive way by recognizing this two-sorted ontology of boundaries. Bona fide boundaries yield a notion of contact that is effectively modeled by classical topology; the analogue of contact involving fiat boundaries calls, however, for a different account, based on the intuition that fiat boundaries do not support the open/closed distinction on which classical topology is based. In the presence of this twosorted ontology it then transpires that mereotopology-typology erected on a mereological basis-is more than a trivial formal variant of classical point-set topology.
\end{abstract}

\section{Parts and Boundaries}

Consider John, the moon, a lump of cheese. These are objects possessed of divisible bulk. They can be divided, in reality or in thought, into spatially extended parts. They have interiors. They also have boundaries, which we can think of (roughly) as infinitely thin extremal slices. The boundary of the moon is the lunar surface. The boundary of John is the surface of his skin.

But what of inner boundaries, the boundaries of the interior parts of things? There are many genuine two-dimensional (sphere- and torus-like) boundaries within the interior of John's body in virtue of the differentiation of this body into organs, cells, and so on. Imagine, however, a spherical ball made of some perfectly homogeneous prime matter. If the possession by an object of genuine inner boundaries presupposes either some interior spatial discontinuity or qualitative heterogeneity, then there is a sense in which there are no boundaries to be acknowledged within the interior of such an object at all.

Yet we do sometimes speak of inner boundaries even in the absence of any corresponding physical discontinuity or qualitative differentiation. Even in 
relation to a homogeneous sphere we can still talk sensibly of its upper and lower hemispheres, its center of mass, and so on. We shall call the inner boundaries involved in such cases fiat boundaries. Inner boundaries involving spatial discontinuity (holes, fissures, slits) or qualitative heterogeneity (of material constitution, texture, electric charge) we shall call bona fide boundaries. $^{1}$

\subsection{Fiat Boundaries and Fiat Objects}

The distinction between fiat and bona fide boundaries applies not only to inner boundaries but to outer boundaries, too. The surfaces of extended objects such as planets or tennis balls are of the bona fide sort. National borders, by contrast, as well as county- and property-lines and the borders of postal and electoral districts, provide examples of outer boundaries of the fiat sort, at least in those cases where they lie skew to any qualitative differentiations or spatial discontinuities (coastlines, rivers) in the underlying territory.

There are different types of fiat boundaries. Thus there are fiat boundaries in the social world-such as those drawn by real estate developers or by international boundary commissions-which can be compared to claims, obligations, and other sorts of social object. They have a quasi-abstract character in the sense that they are relatively isolated from causal change. But they are not completely isolated: there is standardly a point in time at which they begin to exist, and while they exist they may be associated with specific systems of legal or other sorts of sanctions. Further, they manifest a type of generic dependence upon associated beliefs and customs on the part of relevant human beings, so that they may be sustained in being from generation to generation.

There are also non-social fiat boundaries. Consider, for example, the boundaries depicted in atlases of surgical anatomy (between the upper, middle, and lower femur). Fiat boundaries are involved, too, when an individual cognitive agent conceptualizes a sphere as being made of two hemispheres or when a schoolboy draws a circle on a blackboard. And we shall see below that fiat boundaries are involved also in perception. Individual fiats are much more ephemeral than social fiats because they are individually dependent (on these acts, taking place now) rather than generically dependent (on the existence of relevant acts of a certain kind).

There are also fiat boundaries that have a mathematical definition, such as the equator or the center of mass of the moon. In such cases, the question of their ontological status is part-and-parcel of the larger question of the existence and status of mathematical entities in reality.

This terminology was introduced in Smith 1994. See Smith 1995a and Varzi 1997 for some first applications. 
But now, once fiat boundaries have been recognized, it becomes clear that the bona fide-fiat opposition can be drawn not merely in relation to boundaries but in relation to objects also. Examples of bona fide objects are: John, the moon, a lump of cheese. Examples of fiat objects are: Dade County, the State of Wyoming, the North Sea (whose objectivity, as Frege writes (1884, \$26), "is not affected by the fact that it is a matter of our arbitrary choice which part of all the water on the earth's surface we mark off and elect to call the "North Sea"').

Broadly, it is the drawing of fiat outer boundaries in the spatial realm which yields fiat objects. We say broadly, since there are cases of objects which ought reasonably to be classified as fiat objects whose boundaries involve a mixture of bona fide and fiat elements. The shores of the North Sea are bona fide boundaries; but we conceive the North Sea as a fiat object nonetheless, because where it abuts the Atlantic it has a boundary of a nonbona fide sort. Moreover, there are normally perfectly good reasons - reasons of topography, economy, or military strategy-why these and those fiat objects are created rather than others. Fiat objects thus owe their existence not exclusively to human fiat: real properties of the underlying factual material are involved also. These both enable the drawing of fiat borders and constrain the types and location of fiat borders which can be drawn.

Nor are there only two-dimensional fiat objects correlated with selected regions on the surface of the globe. Examples of three-dimensional fiat objects are provided by the subterranean volumes of land to which mineral rights have been assigned, and also by the sectors and corridors in space established for the purposes of air traffic control. These may be quite complicated three-dimensional worms; they may intersect each other and they may have holes. Moreover, insofar as an object whose boundary is not entirely of the bona fide variety counts as a fiat object, many ordinary entities will also qualify as three-dimensional fiat objects. A mountain, a bay, the branches of a tree, or the stem of a champagne glass are all fiat objects in this sense.

Finally, all the examples of fiat objects mentioned so far are cases where proper parts are delineated or carved out by fiat on the surfaces or within the interiors of larger bona fide wholes. However, while we can reasonably assume that all bona fide objects are connected, fiat objects may be scattered, as in the case of Polynesia, the Polish nobility, the constellation Orion. Following Meinong (1899), we might refer to such entities as 'higher-order' (fiat) objects. Objects of this sort may themselves be unified together into further fiat objects (say: the Union of Pacific Island Nations). The fiat boundaries to which higher-order fiat objects owe their existence are the mereological sums of the (fiat and bona fide) outer boundaries of their respective lowerorder constituents. 


\subsection{Boundaries and Cognition}

One reason for resisting scepticism in face of the fiat world turns on the fact that people kill each other over fiat borders, and they give their lives to defend them. Even in times of peace there are entire industries (of real estate law, cadastral registration, land surveying) which are devoted to their maintenance. But are the geographical and political examples upon which our remarks have been concentrated so far truly of central ontological importance? Here we shall content ourselves with considering what might be the justification for awarding the categories of fiat boundaries and fiat objects a crucial organizing role in our categorial scheme.

To grasp the theoretical significance of the examples mentioned thus far, we must consider certain topological peculiarities of fiat boundaries. Consider what happens when two political entities (nations, states, counties) lie adjacent to one another. The entities in question may be said to share a common boundary. This sharing or coincidence of spatial boundaries is, we want to claim, a peculiarity of the fiat world: it has no analogue in the world of bona fide entities. To see this, it may suffice to imagine that two bodies, say John and Mary, should converge upon each other for a period of time, for example in shaking hands or kissing. Physically speaking, as we know, a complicated story has to be told in such cases as to what happens in the area of apparent contact of the two bodies, a story in terms of sub-atomic particles whose location and whose belongingness to either one or the other of the two bodies may be only statistically specifiable. As far as bona fide outer boundaries are concerned, however, no genuine contact or coincidence of boundaries between John and Mary is possible at all. Certainly every genuine kiss involves real physical phenomena (relating to surface tension, fluid exchange, compacting of molecules) as well as associated real psychological phenomena (of tactile and emotional feeling, etc.). But these are merely such as to provide an appropriate real basis for the sorts of fiat demarcations which are effected when we use the simple terms of natural language to describe the relevant larger-scale phenomena.

We apprehend the world as consisting of (fleets of) ships, (pairs of) shoes and (ounces of) sealing wax, and in each case fiat boundaries are at work in articulating the reality with which we have to deal. Natural language contributes to the generation of fiat boundaries also through the opposition between mass nouns (such as 'water') and count nouns (such as 'person'). A hungry carnivore points towards the cattlefield and pronounces 'There is cow over there'. How does this pronouncement differ, in its object, from 'There are cows over there'? Not, certainly, in the underlying real bovine material. Rather, it differs in virtue of the different sorts of boundary that are imposed upon this material in the two cases. 
In fact, our cognition of external reality involves the systematic imposition of boundaries of many different sorts, including fiat boundaries which may be more or less ephemeral. ${ }^{2}$ One important motor for the drawing of ephemeral fiat boundaries is perception, which as we know from our experience of Seurat paintings has the function of articulating reality in terms of sharp boundaries even when such boundaries are not genuinely present in the autonomous (which is to say mind-independent) physical world. Holes, dents, bumps and protrusions are also to be counted as fiat objects in this sense. Consider the Grand Canyon. That part of its boundary which serves as (virtual) lid, separating it from the body of air above it, is a fiat boundary: it corresponds to no physical discontinuity. ${ }^{3}$

We have thus far been leaving out of account borders commonly conceptualized in terms of zones rather than of abstract geometrical lines-objects of the types depicted for example on weather maps. Here, too, the notion of fiat boundary can be brought into play. Consider, for example, deserts, valleys, dunes, clouds. Such objects seem to be delineated not by crisp outer boundaries but by imprecise, boundary-like regions. This should not, however, be taken to imply that the underlying reality is in some respects ultimately vague - that we should allow for a distinction between crisp and genuinely scruffy (fuzzy, hazy, indeterminate) denizens of reality, as some have urged. ${ }^{4}$ Rather, vagueness is a conceptual and semantic matter that pertains ontologically only to the fiat world. If you point to an irregularly shaped protuberance in the sand and say 'dune', then the correlate of your expression is a fiat object whose constituent unitary parts are comprehended (articulated) through the concept dune. The vagueness of the concept is responsible for the way in which the referent of your expression is picked out. Likewise, when you baptize a piece of land 'Mount Everest', the referent of the term is vaguely fixed, and in this sense Mount Everest is a vague fiat object. But no part or feature of bona fide reality is in and of itself vague. Each one of a large variety of slightly distinct aggregates of molecules has an equal claim to being the referent of your newly introduced name. And each such aggregate is precisely determinate. ${ }^{5}$

\section{Problems of Contact and Separation}

A fundamental question arises as soon as boundaries are taken seriously into account. A boundary demarcates two entities, or two parts of the same entity,

The theory of fiat boundaries is thus a contribution to the formal theory of the commonsense world of the sort set out in Hobbs and Moore 1985, and it supplements Stroll 1988. See Smith 1995b for a general survey.

3 See Varzi 1996b. See also Smith and Varzi 1999 for applications of this idea to the formal ontology of niches and environmental settings.

See for instance Tye 1990.

Thus Quine 1985, pp. 167-68. 
which are then said to be in contact with each other. How is this relation of contact to be explained?

We have seen that as far as bona fide outer boundaries are concerned, our everyday intuitions on these matters are in need of revision. For both physical and mathematical reasons, the surfaces of distinct physical bodies cannot be in contact, though two bodies may of course be so close to each other that they appear to be in contact to the naked eye. (This is why natural language does not distinguish between true topological contact-or connection, as we may also say-and mere physical closeness.) Yet we wish, surely, to hold on to the idea that there is genuine contact between John's head and the rest of his body. And this may seem to cause problems. Shall we say, following Bolzano (1851), that contact is only possible if one of these two entities (the head or the rest of the body) lacks a boundary of its own? Or shall we rather follow Brentano (1976) and maintain that there are here two boundaries (one belonging to the head and one to the body) which share exactly the same location?

Imagine ourselves proceeding along a line through the middle of a disk that is divided into two precisely symmetrical regions. What happens as we pass the boundary between the two? Do we pass through a last point $x$ in the first and a first point $y$ in the second? Clearly not, given the density of the continuum; for then we should have to admit an indefinite number of further points between $x$ and $y$ which would somehow be in neither region. To acknowledge the existence of just one of $x$ and $y$ but not the other, however, as is dictated by the standard mathematical treatment of the continuum, would be to countenance a peculiar privileging of one of the two regions over the other, and such an unmotivated asymmetry can surely be rejected as a contravention of the principle of sufficient reason.

The difficulty in providing satisfactory answers to issues such as this has served to impugn the realist attitude towards boundaries quite generally, and these have accordingly been assigned to almost total oblivion in the history of metaphysics. Here, however, we shall argue that this is a mistake stemming precisely from the failure to appreciate the distinction between fiat and bona fide boundaries.

\subsection{The Open/Closed Opposition}

A more precise statement of the standard argument against a realist ontology of boundaries is as follows: (1) Admitting boundaries implies a distinction between closed and open entities-i.e., between entities that do and entities that do not include their boundaries among their constituent parts. ${ }^{6}$ But (2)

6 An entity may include parts of its boundary, but not the whole, and thereby qualify as partly closed and partly open. In the following we shall ignore the complications that arise in such cases and speak of partly open objects as being open simpliciter. 
the open/closed distinction is in this respect counterintuitive: it seems to imply that, if a body is divided in half, then we must be left with one part that is closed and another that is open. Thus (3) we must do without boundaries (and without the open/closed distinction) and regard talk of boundaries as a mere façon de parler about other things-for example about infinite series.?

We shall object to both premisses of this argument. First, premiss (1) corresponds to the assumption that an ontology of boundaries must be based on ordinary topology. We shall argue that this assumption is indeed correct for bona fide boundaries. However, fiat boundaries-and the analogue of contact which they involve-call for a different sort of topology which dispenses with the open/closed distinction. Where fiat boundaries are present, even if only as segments of larger boundaries which include also bona fide portions, the usual trichotomy: open, closed, partly-open-and-partly-closed, does not apply.

Second, the open/closed distinction is not in and of itself counterintuitive. Indeed, in some cases it seems quite reasonable: ordinary material objects are naturally the owners of their boundaries (their surfaces, in effect), and there is nothing counterintuitive in the thought that the environments in which objects are embedded are open. ${ }^{8}$ Thus, premiss (2) is also ill-grounded.

Consider, however, the alleged difficulty with the phenomenon of separation (the mirror image of the phenomenon of contact). Suppose we dissect a solid sphere. This creates two half-spheres. Is one closed and the other open? This very question arises, we insist, only on the basis of an incorrect model of what happens topologically when a process of cutting takes place. ${ }^{9}$ Such a process does not reveal additional surfaces which have been trapped, as it were, inside the sphere until the cutting took place. Rather, the extant outer surface of the sphere is progressively deformed. Think of a splitting oil drop. The drop grows longer and, as it grows, the middle part shrinks and gets thinner and thinner. Eventually the right and left portions split and we have two drops, each with its own complete boundary. A long, continuous process suddenly results in an abrupt topological change. There was one drop; now there are two. And so in the case of the dissected sphere. There was one surface, and now there are two.

This account reduces the problem of cutting to that of separating two spheres that are connected by one tiny point. But does this really solve the problem? Where does this one point belong-to the left sphere or the right

7 This argument is rooted in the work of Whitehead 1929 and is exemplified in the recent literature by Gotts et al. 1996. For a survey of related positions see Varzi 1997.

8 On the idea that material objects have open complements, see also Asher and Vieu 1995 and Smith and Varzi 1999. Casati and Varzi 1994 argue that holes, in particular, are bounded from the outside: the boundary of a hole is the surface of its material host. For other families of examples see Jackendoff 1991. This point expands on an argument put forward in Varzi 1997, \$7. 
one? There is indeed something deeply problematic about the point of separation. However, this is true of every topological change. Consider:

1) Two drops of oil move toward each other until they come into contact. An abrupt change takes place: the topology of the overall configuration is suddenly altered. Two surfaces merge. Two drops become one.

2) A worm drills a hole in a log of wood and breaks through to the other side. Once again, an abrupt change takes place at the termination of such a process: a sphere becomes a doughnut; the topology of the object undergoes a qualitative transformation. Or consider a piece of soft plasticine (a mushy blob) through which you make a perforation by slowly pressing your finger: there then occurs a constant elastic deformation which terminates when your finger-mirabile dictu-breaks through to the other side.

3) A bed of coral starts growing a "finger" somewhere. The finger continues to grow until it eventually comes round to meet the main body again, forming a sort of handle. At the instant that it does so, the topology of the object changes: where once we had a sphere, now we have a torus.

All these cases involve something genuinely problematic. But this reffects only the fact that topological change marks one point at which common sense reaches the limits of its theoretical competence, and we can of course provide accounts of these same phenomena also from the perspective of physical science. We then discover that macroscopic physical objects are not continuous and that they do not have boundaries of the sort countenanced by common sense at all. When we move to adopt the physical perspective, then our topological problems disappear. You make a tunnel by removing the last layer of molecules or by augmenting their relative distance (and when you start having a tunnel may well be a vague matter). You split two things when you pull apart the last two molecules or atoms in such a way as to create a gulf between them. No mystery remains. But what follows from this? Not that we should give up talk of macroscopic boundaries altogether; for whatever is to be said about the ontology of the physical world, one still needs such talk when it comes to the fiat entities carved out by ordinary discourse and to the spatial regions which these occupy.

\subsection{Coincidence of Fiat Boundaries}

As long as we confine ourselves to a topological model, then, cutting a solid object does not bring surfaces to light. Thus the demarcation puzzle-the puzzle of providing a principled way of determining, when a body is divided in half, which half is open and which is closed-does not arise, and this blocks the argument against bona fide boundaries. However, suppose we do not actually cut the object. Suppose we simply conceptualize a fiat boundary separating it into two halves. Is this not enough to give rise to the demarcation puzzle? Which half gets to own the boundary? To which hemisphere 
does the equator belong? Or think of the Mason-Dixon line separating Maryland and Pennsylvania. There is no fact of the matter that can support the ownership of a border such as this by one piece of land rather than another. Yet we cannot simply say that the borders are unowned: the States of the Union use up the whole territory-no boundaries can be left as thin slices between them.

It is here that the peculiarity of fiat boundaries comes into play. Fiat boundaries do not introduce any physical discontinuity. But nor are they merely potential entities: it is not that they can come to constitute a discontinuity by having their status changed from fiat to bona fide. Certainly it may be possible, where one has a fiat boundary in a physical object, to generate a bona fide boundary in the corresponding place, e.g., by cutting. But as we have seen, the fiat boundary does not then become a bona fide boundary: no pre-existent inner surface is brought to light by a process of cutting. Likewise, some national borders will in course of time come to involve boundarymarkers (barbed wire fences and the like) which will tend in cumulation to replace what is initially a pure fiat boundary with something more substantial. But this is not a process of transformation. The categorial distinction between fiat and bona fide boundaries is absolute.

How, then, do we account for the ownership of fiat boundaries as such? Does the equator belong to the Northern or to the Southern hemisphere? Our answer-in fact the only answer which remains as a possible option-is that it belongs to both. Or, more precisely, each hemisphere has its own equator, and the two equators coincide (i.e., they have the same spatial location).

This suggestion draws on Brentano. As pointed out by Chisholm, topological connection is to be explained, for Brentano, not via the open/closed opposition, but in terms of boundary coincidence. ${ }^{10}$ Brentano in fact regards the possibility of coincidence as a distinguishing feature of all boundaries. However, we do not need to embrace this view as a general theory of boundaries, for we have seen that the demarcation puzzle is not a problem when the demarcation involved is due to a genuine qualitative discontinuity (a bona fide boundary). Rather, we want to regard Brentano's theory as a theory of what goes on when objects or their parts are separated merely by fiat. It is here that coincidence relations become relevant. We can speak of the Mason-Dixon line as the border between Maryland and Pennsylvania. But this single border is to be recognized as being made up of two parts, two perfectly coinciding fiat boundaries bounding Maryland and Pennsylvania, respectively.

One derivative advantage of this doubling up is that it also yields the possibility of admitting asymmetric boundaries, boundaries which bound

10 See Brentano 1976, p. 41; see also Brentano 1924, pp. 357f. Brentano's theory of the continuum has been examined by Roderick Chisholm in a number of papers; see especially 1984, 1992/3. Smith 1997 provides a detailed formal theory. 
their objects in certain directions only and not in others. This is the case where only one of the two potentially co-located fiat boundaries is actually present as, for example, at the old border between the German Democratic and Federal Republics.

\section{Towards a Formal Theory}

We thus have two complementary boundary theories. The first, more classical theory, holds that genuine contact is only possible between two entities one of which is open in the contact area and the other closed. The second yields a quite new form of topology. It turns on the contrary insight, according to which what is characteristic of a continuum is the possibility of a coincidence of boundaries. The two theories are not in complete disagreement. Both bona fide and fiat boundaries share a fundamental property: they are ontologically parasitic on (i.e., cannot exist in isolation from) their hosts, the entities they bound. This is a common feature that a comprehensive treatment of boundary phenomena should emphasize. In providing a more precise formulation of the two theories, we shall therefore start with their common core and then move on to the two needed supplements.

\subsection{The Common Core}

The fundamental ontological property of boundaries was given a clear formulation by Brentano himself (who in turn elaborated on Aristotle's sketchy remarks in the Physics and the Metaphysics): if something continuous is a mere boundary, then it can never exist except in connection with other boundaries and except as belonging to a continuum of higher dimension. ${ }^{11}$ There are, in reality, no isolated points, lines, or surfaces. Boundaries are, in Chisholm's terms, dependent particulars. They are in this respect comparable to universal forms or abstract structures (for example to the structure of a molecule which exists only as belonging to a given concrete instance). This must be said of all boundaries, including those which possess no dimension at all, such as spatial points: a cutting free from everything that is continuous and extended is for them, too, impossible.

Dependent or parasitic entities license certain sorts of ontological inference (if there is a boundary/structure/universal having these and those properties, then there is a host having these and those properties). We cannot infer to any specific host, however. Thus it cannot be said of any definite continuum that a boundary is dependent on it. That which a boundary is dependent on can be designated rather only via a general term: what is required by a boundary is, Brentano says, "not this or that particular continuum, but any continuum of the appropriate kind" (1933, p. 56; translation corrected). For while no boundary can exist without being connected with a continuum, "there is no 
specifiable part, however small, of the continuum, and no point, however near it may be to the boundary, which is such that we may say that it is the existence of that part or of that point which conditions the boundary." (Ibid.) In short, the continuum is specifically dependent on its boundary, but the boundary is not in this same sense dependent on its continuum; it is only generically so. ${ }^{12}$

It is impossible to do justice to these distinctions without resorting in some way to modal notions. However, we shall attempt in what follows to embed the dependent nature of boundaries at least into a basic non-modal mereological (more generally, mereotopological) framework. Our aim will be illustrative, so we shall not be too concerned with the question of what sort of formal mereological theory is most adequate for this purpose. We shall, however, try to be rather specific as concerns the question of how such a mereological background can be integrated with a theory of boundaries of the bona fide and fiat sorts. ${ }^{13}$

\subsection{Mereology}

Mereology is the formal theory of parthood relations: of the relations of part to whole and the relations of overlap within a whole. For simplicity, we shall assume a standard mereological apparatus constructed around the primitive is part of, which we symbolize as ' $\leq$ '. ${ }^{14}$ (We take ' $x \leq y$ ' to be true when $x$ is any sort of part of $y$, including an improper part, so that $x \leq y$ will be consistent with $x$ 's being the same as $y$.) If we define overlap in the usual way:

$$
\mathrm{D} \leq 1 \quad \mathrm{O}(x, y):=\exists z(z \leq x \wedge z \leq y)
$$

then the axioms for this mereological background can be formulated as follows: ${ }^{15}$

$\mathrm{A} \leq 1 \quad x \leq x$

$\mathrm{A} \leq 2 x \leq y \wedge y \leq x \rightarrow x=y$

$\mathrm{A} \leq 3 x \leq y \wedge y \leq z \rightarrow x \leq z$

\footnotetext{
12. On boundaries as dependent particulars see Chisholm 1984. See Smith 1992, $\$ 10$, for further discussion of the generic dependence of boundaries upon their hosts.

13 Further formal details are provided in Smith 1993, 1997 and Varzi 1996a, 1997.

14 For an introduction to standard mereology and its variants, see Simons 1987.

15 Here and in the sequel initial universal quantifiers are to be taken as understood, and variables are to be conceived as ranging over all spatial entities, both extended and nonextended. Moreover, our axioms are to be read synchronically: thus, $A \leq 5$ is to guarantee the existence of a fusion of all entities that satisfy $\phi$ at any given time $t$, but not the crosstemporal fusion of all entities that satisfy $\phi$ at some time or other.
} 


$$
\mathrm{A} \leq 5 \exists x(\phi x) \rightarrow \exists y \forall z(\mathrm{O}(y, z) \leftrightarrow \exists x(\phi x \wedge \mathrm{O}(x, z)))
$$

Thus, parthood is a reflexive, antisymmetric, and transitive relation, a partial ordering. In addition, $\mathrm{A} \leq 4$ ensures that parthood is extensional, whereas the schema $A \leq 5$ guarantees that for every satisfied property or condition $\phi$ (i.e., every condition $\phi$ that is true of at least one object) there exists an entity, the sum or fusion (unique by $A \leq 4$ ), consisting precisely of all the $\phi e r s$. This entity will be denoted by ' $\sigma x(\phi x)$ ' and is defined as follows: ${ }^{16}$

$$
\mathrm{D} \leq 2 \quad \sigma x(\phi x):=\mathfrak{t} y \forall z(\mathrm{O}(y, z) \leftrightarrow \exists x(\phi x \wedge \mathrm{O}(x, z)))
$$

With the help of this operator, other useful notions are easily defined. In particular, we can define the operators of mereological sum $(+), \operatorname{product}(\times)$, and complement (-):

$$
\begin{aligned}
& \mathrm{D} \leq 3 x+y:=\sigma z(z \leq x \vee z \leq y) \\
& \mathrm{D} \leq 4 x \times y:=\sigma z(z \leq x \wedge z \leq y) \\
& \mathrm{D} \leq 5-x:=\sigma z(\neg \mathrm{O}(z, x)) .
\end{aligned}
$$

\subsection{The Theory of Bona Fide Boundaries}

Let us now proceed to the formulation of the basic principles for boundaries. We shall begin with the theory of bona fide boundaries, which effectively corresponds to an ontology based on ordinary, Bolzanian topology; we shall then move on to the Brentanian theory for fiat boundaries.

We shall symbolize the primitive boundary relation by ' $\mathrm{B}$ ', reading ' $\mathrm{B}(x$, $y)$ ' as " $x$ is a bona fide boundary for $y$ ". We say boundary for, rather than boundary of, to allow for boundaries that are not maximal (edges, corners, parts of surfaces). The notion of a maximal bona fide boundary of $x$ is then immediately defined, using $\mathrm{A} \leq 5$, as the sum of all bona fide boundaries for $x$ :

$\mathrm{DB} 1 \mathrm{~b}(x):=\sigma z(\mathrm{~B}(z, x))$.

Of course, this is a partial operator that need not be defined for all values of ' $x$ '. For instance, if $x$ is the State of Wyoming, then $x$ has no bona fide boundaries and $b(x)$ is undefined. Likewise, an arbitrary cube within the interior of a homogeneous sphere has no maximal boundary in the sense of DB1.

16 We assume the definite descriptor ' $i$ ' to be contextually defined in standard Russellian fashion. 
Let us now define the (partial) operator for topological closure in the obvious way:

DB2 $\mathrm{c}(x):=x+\mathrm{b}(x)$.

Then the basic postulates for a topology based on bona fide boundaries can be given by mereologizing the standard Kuratowski axioms for closure operators: $:^{17}$

$$
\begin{aligned}
& \text { AB1 } x \leq \mathrm{c}(x) \\
& \text { AB2 } \mathrm{c}(\mathrm{c}(x)) \leq \mathrm{c}(x) \\
& \mathrm{AB} 3 \mathrm{c}(x+y)=\mathrm{c}(x)+\mathrm{c}(y) .
\end{aligned}
$$

(In view of $\mathrm{DB} 2$, axiom $\mathrm{AB} 1$ is actually derivable from $\mathrm{A} \leq 1$ and $\mathrm{A} \leq 3$.)

This yields a straightforward reformulation of much of standard topology based on extensional mereology instead of set theory. In particular, it implies that bona fide boundaries are always transitive and dissective:

$\mathrm{TB} 1 \mathrm{~B}(x, y) \wedge \mathrm{B}(y, z) \rightarrow \mathrm{B}(x, z)$

TB2 $x \leq y \wedge \mathrm{B}(y, z) \rightarrow \mathrm{B}(x, z)$.

They are also symmetric, in the sense that a bona fide boundary for a given entity is also a bona fide boundary for that entity's complement:

TB3 $\mathrm{B}(x, y) \rightarrow \mathrm{B}(x,-y)$.

The symmetry of ' $\mathrm{B}$ ' allows us to define the relation of (bona fide) connection as the sharing of a common part or boundary, in the following sense:

DB3 $\mathrm{C}(x, y):=\mathrm{O}(x, y) \vee \mathrm{O}(\mathrm{c}(x), y) \vee \mathrm{O}(x, \mathrm{c}(y))$.

Accordingly, if we define contact as external connection, i.e., connection without overlap:

$\mathrm{DB} 4 \mathrm{EC}(x, y):=\mathrm{C}(x, y) \wedge \neg \mathrm{O}(x, y)$,

and if we define closed entities in the obvious way,

DB5 $\mathrm{Cl}(x):=x=\mathrm{c}(x)$,

17 These axioms are to be understood as holding whenever ' $c$ ' is defined for the relevant arguments. See Smith 1993 and Pianesi and Varzi 1996 for alternative axiomatizations. 
then we can immediately infer from the above that two entities can be in contact only if one of them is not closed:

$\mathrm{TB} 4 \mathrm{EC}(x, y) \rightarrow(\mathrm{Cl}(x) \rightarrow \neg \mathrm{Cl}(y))$.

Thus, contact between John and Mary is simply not possible if this is understood in terms of external connection. This is in agreement with physics and with ordinary topology. In addition, however, it also follows that, so long as we have at our disposal only the notion of bona fide contact, John's (undetached) head is not in contact with John's body-for there is no bona fide boundary connecting these two respective parts. This marks the point where we need to depart from ordinary topology and move to an account in terms of fiat boundaries.

Reference to fiat boundaries will also be needed to capture the idea that bona fide boundaries are dependent particulars. This thesis-which stands opposed to the set-theoretic conception of boundaries as, effectively, sets of independent points, each one of which could exist though all around it be annihilated-has a number of possible interpretations. ${ }^{18}$ One general statement of the dependence thesis would assert that the existence of any boundary is such as to imply the existence of some entity of higher dimension which it bounds. Here, though, we must content ourselves with the formulation of a simpler thesis to the effect that every boundary is such that we can find an entity which it bounds and which is not itself a boundary (i.e., which has an interior). To this end, we may define the relational predicate of interior parthood:

$\operatorname{DB} 6 \operatorname{IP}(x, y):=x \leq y \wedge \forall z(\mathrm{~B}(z, y) \rightarrow \neg \mathrm{O}(x, z))$.

Obviously, boundaries have no interior parts:

TB5 $\mathrm{B}(x, y) \rightarrow \neg \mathrm{IP}(z, x)$.

We define also, for convenience, a monadic predicate of bona fide boundary:

$\operatorname{DB} 7 \operatorname{Bd}(x):=\exists y \mathrm{~B}(x, y)$.

We could then provide a first formulation of the dependence thesis for bona fide boundaries:

$\mathrm{AB} 4 \mathrm{Bd}(x) \rightarrow \exists y \exists z(\mathrm{~B}(x, y) \wedge \mathrm{IP}(z, y))$.

This is not very strong, however. For as it turns out, AB4 is always trivially satisfied by choosing $z$ open (i.e., such that $\operatorname{IP}(z, z)$ ) and setting $y$ equal to

18 See Smith 1997 for an extended treatment. 
the scattered object $x+z$. A dependence thesis of the required strength must therefore impose on $y$ in $\mathrm{AB} 4$ at least the additional requirement of being selfconnected (being all of a piece). To express this requirement, however, we need to have at our disposal the resources of a topology of fiat boundaries.

\subsection{The Theory of Fiat Boundaries}

Let us use ' $\mathrm{B}$ '' as a primitive for fiat boundaries, reading ' $\mathrm{B} *(x, y)$ ' as ' $x$ is a fiat boundary for $y$ ". A bona fide boundary for $y$ is in every case also a boundary for the complement of $y$ (TB3), though it belongs as part only to one or the other of these. In contrast, the main distinguishing feature of fiat boundaries is that they are necessarily parts of the entities they bound. A fiat boundary for $y$ is in every case a part of $y$ : this is the first axiom for ' $\mathrm{B}$ '?

$$
\mathrm{AB}^{* 1} \mathrm{~B}^{*}(x, y) \rightarrow x \leq y .
$$

Note that parthood, here, embraces not merely physical but also social objects. When, in 1922, the British High Commissioner Sir Percy Cox drew lines on a map marking the boundaries of Iraq, Kuwait, and Saudi Arabia, he thereby added new non-physical ingredients to the world.

It follows from $\mathrm{AB}^{*} 1$ that there is no significant analogue of the closure operator ' $c$ ' in the fiat world: the sum of an object with its fiat boundaries is in every case just the object itself. There is therefore no significant counterpart to the Kuratowski axioms in the theory of fiat boundaries. It also follows from $\mathrm{AB}^{*} 1$ that ' $\mathrm{B}$ '' does not satisfy the analogue of TB3: fiat boundaries are not symmetric (and so we might, in certain circumstances, talk of "oriented boundaries"). However, we can assume dissectivity (the analogue of TB2):

$$
\mathrm{AB}^{*} 2 x \leq y \wedge \mathrm{B}^{*}(y, z) \rightarrow \mathrm{B}^{*}(x, z),
$$

whence transitivity (the analogue of $\mathrm{TB} 1$ ) follows immediately:

$$
\mathrm{TB}^{* 1} \quad \mathrm{~B} *(x, y) \wedge \mathrm{B}^{*}(y, z) \rightarrow \mathrm{B}^{*}(x, z) .
$$

Thus, by $\mathrm{AB}^{*} 2$ every linear segment of the border of Wyoming is a fiat boundary for Wyoming; and by $\mathrm{TB}^{*} 1$ any point serving as a boundary of a segment of the border of Wyoming is a boundary point for Wyoming itself.

We can also formulate the fiat analogue of our rudimentary version of the dependence thesis expressed by $\mathrm{AB} 4$. To this end, let us define the fiat analogues of the predicates 'Bd' and 'IP' introduced in DB6 and DB7:

$$
\begin{array}{ll}
\mathrm{DB}^{*} 1 & \mathrm{IP}^{*}(x, y):=x \leq y \wedge \forall z\left(\mathrm{~B}^{*}(z, y) \rightarrow \neg \mathrm{O}(x, z)\right) \\
\mathrm{DB}^{*} 2 & \mathrm{Bd}^{*}(x):=\exists y \mathrm{~B}^{*}(x, y) .
\end{array}
$$


Clearly, we must assume the analogue of TB5: fiat boundaries are boundaries; hence they have no (fiat) interior parts:

$$
\mathrm{AB}^{*} 3 \quad \mathrm{~B}^{*}(x, y) \rightarrow \neg \mathrm{IP} *(z, x)
$$

Then the fiat counterpart of AB4 becomes:

$$
\mathrm{AB}^{*} 4 \quad \mathrm{Bd}^{*}(x) \rightarrow \exists y \exists z\left(\mathrm{~B}^{*}(x, y) \wedge \operatorname{IP}^{*}(z, y)\right) .
$$

We shall see shortly how this thesis can be strengthened to avoid the sort of trivialization already mentioned in connection with AB4.

In order now to characterize the relation between John's head and John's body (the relation of connection by fiat boundary), we rely on the relation of coincidence, which we symbolize by ' $\approx$ '. This relation is to be understood intuitively as obtaining between two entities $x$ and $y$ just in case they share the same spatial location. We shall assume here that material objects cannot coincide in this sense with other material objects. But fiat boundaries, because they are not possessed of divisible bulk, do not occupy (fill out) the space where they are located; hence they can be perfectly co-located one with another. ${ }^{19}$

On this interpretation, coincidence is clearly an equivalence relation, i.e., a reflexive, symmetric, and transitive relation: ${ }^{20}$

$$
\begin{aligned}
& \mathrm{A} \approx 1 \quad x \approx x \\
& \mathrm{~A} \approx 2 \quad x \approx y \rightarrow y \approx x \\
& \mathrm{~A} \approx 3 \quad x \approx y \wedge y \approx z \rightarrow x \approx z .
\end{aligned}
$$

To these axioms we add two postulates to the effect that coinciding entities have coinciding parts and are closed under arbitrary sums:

$$
\begin{array}{ll}
\mathrm{A} \approx 4 & x \leq y \wedge y \approx z \rightarrow \exists w(w \leq z \wedge x \approx w) \\
\mathrm{A} \approx 5 \quad \exists y(\phi y) \wedge \forall y(\phi y \rightarrow x \approx y) \rightarrow x \approx \sigma y(\phi y) .
\end{array}
$$

Thus, in particular, if $x$ coincides with two entities $y$ and $z$, then it coincides also with the sum of $y$ and $z$.

We are finally ready to define Brentanian connection-connection by fiat boundary. The idea is simply that this form of connection obtains between

19 On the difference between location and occupation, see Casati and Varzi 1996, 1999.

20 Our axioms for ' $=$ ' are adopted from Smith 1997. They differ slightly from those given by Chisholm, who takes coincidence to pertain exclusively to boundaries. In particular, reflexivity and transitivity do not hold unrestrictedly in Chisholm's system (see his 1984). 
two adjacent entities, for example Germany and France, whenever their fiat boundaries coincide at least in part:

$$
\mathrm{DB}^{*} 3 \quad \mathrm{C}^{*}(x, y):=\mathrm{O}(x, y) \vee \exists z \exists w\left(\mathrm{~B}^{*}(z, x) \wedge \mathrm{B}^{*}(w, y) \wedge z \approx w\right) .
$$

If $x$ and $y$ overlap then they are both $\mathrm{C}$-connected and $\mathrm{C}^{*}$-connected. The difference between the two relations comes to light in the case of entities that do not overlap. If the boundary through which $x$ and $y$ are connected is a bona fide boundary, i.e., if they are externally C-connected in the sense of DB4, then this boundary bounds one entity from the inside and the other from the outside. (See again TB4, which effectively represents the Bolzanian view of contact.) If, by contrast, the boundary through which $x$ and $y$ are connected is a fiat boundary, i.e., if they are externally $\mathrm{C}^{*}$-connected:

$\mathrm{DB}^{* 4} \quad \mathrm{EC}^{*}(x, y):=\mathrm{C}^{*}(x, y) \wedge \neg \mathrm{O}(x, y)$

then each entity is bounded by its own fiat boundary. It is in this sense that the head of John is in contact with the rest of John's body.

\subsection{Dependence}

At this point we can complete our account of the central intuition that all boundaries (whether fiat or bona fide) are dependent entities. We have seen that $\mathrm{AB} 4$ and $\mathrm{AB} * 4$ do not fully succeed in this respect, and that a predicate of self-connectedness is needed to rule out trivial hosts. In ordinary topology, an entity is said to be self-connected if it does not amount to the sum of two disconnected parts. In the present context, however, the relevant notion of connection is not ' $C$ ' (bona fide connection), for we certainly want to say that John's body and his undetached head form a connected sum. Rather, we must rely on the fiat variety of connection, ' $\mathrm{C}^{*}$ '. A self-connected entity is one all of whose parts are separated at most by fiat:

$$
\mathrm{DB}^{*} 5 \quad \mathrm{Cn}^{*}(x):=\forall y \forall z\left(x=y+z \rightarrow \mathrm{C}^{*}(y, z)\right) \text {. }
$$

We can then amend $\mathrm{AB} 4$ and $\mathrm{AB}^{*} 4$ to the following theses affirming, for connected boundaries, the existence of connected wholes which they are boundaries for:

$$
\text { AB5 } \operatorname{Bd}(x) \wedge \mathrm{Cn}^{*}(x) \rightarrow \exists y \exists z\left(\mathrm{Cn}^{*}(y) \wedge \mathrm{B}(x, y) \wedge \mathrm{IP}(z, y)\right)
$$

$$
\mathrm{AB}^{*} 5 \quad \mathrm{Bd}^{*}(x) \wedge \mathrm{Cn} *(x) \rightarrow \exists y \exists z\left(\mathrm{Cn}^{*}(y) \wedge \mathrm{B}^{*}(x, y) \wedge \mathrm{IP}^{*}(z, y)\right)
$$

Thus, through the notion of self-connectedness, the theory of bona fide boundaries presupposes the theory of fiat boundaries. This implies, surprisingly, a central role for the fiat world even in matters of bona fide ontology. 
It implies also a hitherto unrecognized feature of classical mereotopology, the formal implications of which have still to be examined.

\section{Concluding Remarks}

Let us conclude by underlining again the main points of our account. First, we have argued that the notion of a boundary must play a fundamental role in a categorial scheme that aims at being both realistic and non-reductionist. True, from the perspective of the physical sciences ordinary physical objects are not continuous and they do not have boundaries of the sort countenanced by common sense. But even if naive boundary talk is deemed inadequate with respect to the entities of physics, one still needs boundaries when it comes to the fiat objects grasped in ordinary experience.

Second, we have argued that the basic typology of spatial boundaries involves an opposition between bona fide (or physical) and fiat boundaries, the latter being exemplified especially by boundaries induced through human demarcation. The classical metaphysical problems connected with the notions of adjacency, contact, separation, and division can be resolved in an intuitive way by recognizing this bicategorial nature of boundaries. Bona fide boundaries yield a notion of contact that is effectively modeled by classical topology; the analogue of contact involving fiat boundaries calls for a different account, based on the intuition that fiat boundaries do not support the open/closed distinction on which classical topology is based.

Finally, we have seen that mereotopology-topology erected on a mereological basis-is more than a trivial formal variant of point-set topology. Much mereotopological machinery can be interpreted in standard set-theoretic terms. ${ }^{21}$ But the opposition between fiat and bona fide boundaries cannot be modelled in a natural and intuitive fashion within a topology erected on a settheoretic basis. ${ }^{22}$

\section{References}

Asher, N., and Vieu, L., 1995, 'Toward a Geometry of Common Sense: A Semantics and a Complete Axiomatization of Mereotopology', Proceedings of the 14th International Joint Conference on Artificial Intelligence, San Mateo, CA: Morgan Kaufmann, pp. 846-52.

21 See Cohn and Varzi 1998 for details.

22 Early versions of this paper appeared in The Electronic Journal of Analytic Philosophy (May 1997) and in the Proceedings of the Third International Conference on Spatial Information Theory (Springer Lecture Notes in Computer Science 1329, October 1997). The latter contains additional material on the geographical and geopolitical applications of the ideas here presented. We thank the editors for allowing us to make use of some of this material. Thanks are due also to the National Center for Geographic Information and Analysis which has provided valuable support for this work under the terms of the NSF's Project Varenius. 
Bolzano, B., 1851, Paradoxien des Unendlichen, hrsg. von F. Prihonsky, Leipzig: Reclam (English translation by D. A. Steele, Paradoxes of the Infinite, London: Routledge \& Kegan Paul, 1950).

Brentano, F., 1924, Psychologie vom empirischen Standpunkt, 2. Ausgabe, hrsg. von O. Kraus, Leipzig: Meiner (cited according to the English translation by L. L. McAlister, Psychology from an Empirical Standpoint, London: Routledge \& Kegan Paul, 1950).

Brentano, F., 1933, Kategorienlehre, hrsg. von A. Kastil, Hamburg: Meiner (cited according to the English translation by R. M. Chisholm and N. Guterman, The Theory of Categories, The Hague/Boston/London: Nijhoff, 1981).

Brentano, F., 1976, Philosophische Untersuchungen zu Raum, Zeit und Kontinuum, hrsg. von S. Körner und R. M. Chisholm, Hamburg: Meiner (cited according to the English translation by B. Smith, Philosophical Investigations on Space. Time and the Continuum, London: Croom Helm, 1988).

Casati, R., and Varzi, A. C., 1994, Holes and Other Superficialities, Cambridge, MA, and London: MIT Press.

Casati, R., and Varzi, A. C., 1996, 'The Structure of Spatial Location', Philosophical Studies 82, 205-39.

Casati, R., and Varzi, A. C., 1999, Parts and Places. The Structures of Spatial Representation, Cambridge, MA, and London: MIT Press.

Chisholm, R. M., 1984, 'Boundaries as Dependent Particulars', Grazer philosophische Studien 10, 87-95.

Chisholm, R. M., 1992/3, 'Spatial Continuity and the Theory of Part and Whole. A Brentano Study', Brentano Studien 4, 11-23.

Cohn, A. G., and Varzi, A. C., 1998, 'Connection Relations in Mereotopology', in H. Prade (ed.), Proceedings of the 13th European Conference on Artificial Intelligence, Chichester: John Wiley \& Sons, pp. 150-54.

Frege, G., 1884, Die Grundlagen der Arithmetik, Breslau: Köbner (cited according to the English translation by J. L. Austin, Foundations of Arithmetic, Oxford: Basil Blackwell, 1950).

Gotts, N. M., Gooday, J. M., and Cohn, A. G., 1996, 'A Connection Based Approach to Common-Sense Topological Description and Reasoning', The Monist 78, 51-75.

Hobbs, J. R., and Moore, R. C. (eds.), 1985, Formal Theories of the Commonsense World, Norwood: Ablex.

Jackendoff, R., 1991, 'Parts and Boundaries', Cognition 41, 9-45.

Meinong, A., 1899, 'Über Gegenstände höherer Ordnung und deren Verhältnis zur inneren Wahrnehmung', Zeitschrift für Psychologie und Physiologie der Sinnesorgane 21, 182-272.

Pianesi, F., and Varzi, A. C., 1996, 'Events, Topology, and Temporal Relations', The Monist 78, 89-116. 
Quine, W. V. O., 1985, 'Events and Reification', in E. LePore and B. P. McLaughlin (eds.), Actions and Events. Perspectives in the Philosophy of Donald Davidson, Oxford: Blackwell, pp. 162-71.

Simons, P. M., 1987, Parts. A Study in Ontology, Oxford: Clarendon Press. Smith, B., 1992, 'Characteristica Universalis', in K. Mulligan (ed.), Language, Truth and Ontology, Dordrecht/Boston/London: Kluwer, pp. 50-81.

Smith, B., 1993, 'Ontology and the Logistic Analysis of Reality', in N. Guarino and R. Poli (eds.), Proceedings of the International Workshop on Formal Ontology in Conceptual Analysis and Knowledge Representation, Padova: Ladseb-CNR, 51-68; revised version as 'Mereotopology: A Theory of Parts and Boundaries', Data and Knowledge Engineering 20 (1996), 287-304.

Smith, B., 1994, 'Fiat Objects', in N. Guarino, S. Pribbenow, and L. Vieu (eds.), Parts and Wholes: Conceptual Part-Whole Relations and Formal Mereology. Proceedings of the ECAI94 Workshop, Amsterdam: ECCAI, pp. 15-23.

Smith, B., 1995a, 'On Drawing Lines on a Map', in A. U. Frank and W. Kuhn (eds.), Spatial Information Theory. A Theoretical Basis for GIS, Berlin/Heidelberg: Springer-Verlag, pp. 475-84.

Smith, B., 1995b, 'The Structures of the Common-Sense World', Acta Philosophica Fennica 58, 290-317.

Smith, B., 1997, 'Boundaries: An Essay in Mereotopology', in L. H. Hahn (ed.), The Philosophy of Roderick Chisholm (Library of Living Philosophers), Chicago and La Salle, IL: Open Court, pp. 534-61.

Smith, B., and Varzi, A. C., 1999, 'The Niche', Nô̂s 33, 214-38.

Stroll, A., 1988, Surfaces, Minneapolis: University of Minnesota Press.

Tye, M., 1990, 'Vague Objects', Mind 99, 535-557.

Varzi, A. C., 1994, 'On the Boundary Between Mereology and Topology', in R. Casati, B. Smith, and G. White (eds.), Philosophy and the Cognitive Sciences, Vienna: Hölder-Pichler-Tempsky, pp. 423-42.

Varzi, A. C., 1996a, 'Parts, Wholes, and Part-Whole Relations: The Prospects of Mereotopology, 'Data and Knowledge Engineering 20, 259-86.

Varzi, A. C., 1996b, 'Reasoning about Space: The Hole Story', Logic and Logical Philosophy 4, 3-39.

Varzi, A. C., 1997, 'Boundaries, Continuity, and Çontact', Nô̂s 31, 26-58.

Whitehead, A. N., 1929, Process and Reality. An Essay in Cosmology, New York: Macmillan. 